\title{
Fatty Acid-Related Health Lipid Index of Raw and Fried Nile Tilapia (Oreochromis niloticus) Fish Muscle
}

\author{
Aemiro Tadesse Zula (iD) and Derese Tamiru Desta (iD) \\ School of Nutrition, Food Science and Technology Academic Center of Excellence in Human Nutrition, Hawassa University, \\ Awasa, Ethiopia
}

Correspondence should be addressed to Aemiro Tadesse Zula; aemtadzu@gmail.com

Received 1 November 2020; Revised 28 January 2021; Accepted 15 March 2021; Published 20 March 2021

Academic Editor: Constantin Apetrei

Copyright (c) 2021 Aemiro Tadesse Zula and Derese Tamiru Desta. This is an open access article distributed under the Creative Commons Attribution License, which permits unrestricted use, distribution, and reproduction in any medium, provided the original work is properly cited.

\begin{abstract}
Fried food consumption is popular in most parts of the world including Ethiopia. Among many fried products available in Ethiopia, fried fish is most commonly consumed in Hawassa Town due to the easy access to the fish from the lake. Recently, there is growing concern among fryers to recycle the oil while frying fish. However, there is limited evidence about the frying effect on the fatty-acid-related health lipid index of fried fish. Thus, the study was aimed to determine the fatty acid profile and the fattyacid-related health lipid of raw and fried fish. Raw and fried fish were taken from the Hawassa open fish market. Fatty acid profiles were analyzed using a gas chromatography-mass spectrophotometer (GCMS), and the health lipid index was determined by calculation using the recommended formula. JMP pro 13 version software was used for data analysis. Our result showed that raw fish had a high amount of essential fatty acid, nutritive value index, hypocholesterolemic ratio, and peroxidizability index. In contrary to this, the fried fish had a high amount of trans-fatty acids, nonessential fatty acids, atherogenic index, and thrombogenic index. In conclusion, the fried fish loses its fatty-acid-related nutritional quality in uncontrolled frying conditions. Therefore, frying needs to be controlled, as it risks human health otherwise.
\end{abstract}

\section{Introduction}

Fish is a source of essential nutrients and is highly promoted by nutritionists and health-care workers because it has a high amount of double-bonded fatty acids and plays an important role in chronic disease prevention [1]. According to Leung et al. [2], complimentary food including fish for infants is highly recommended. However, according to Gadiraju et al. [3], fish is highly susceptible to safety and quality deterioration, and this leads the fish to lose its nutritional quality due to processing.

Currently, fried food consumption is highly accustomed as it has a good sensory appeal, such as crispy texture, color, and taste [4], and consumption of fried fish has become more common in the world including Ethiopia. Oke et al. [5] stated that changes such as fat absorption, moisture loss, and oxidation reactions occur during frying, and this generates free radicals that affect the safety of fish and considered a risk factor for chronic diseases which can limit the life span of the consumer. Thus, according to FAO [6], a risk inspection and control system should be established for minimizing such problems.

People living around the lake Hawassa (Ethiopia) are highly vulnerable to the health problem associated with fried fish consumption. Around the lake, in the open fish market, three main products are served (fish soup, fried fish, and fish fillet), and Nile tilapia fish is one of the important commercial fish at lake Hawassa [7].

The main fish processing method across Hawassa open fish markets is frying, and no studies have been conducted about identifying the impact of frying on the nutritional quality of fish muscle from the Hawassa open fish market; thus, it is necessary to study the fatty-acid-related nutritional quality of fish muscle, and this would in turn help in identifying the key risks and intervene across the fish value chain, mainly frying in the open fish market. Therefore, the 
current study aims to characterize the fatty acid profile of raw and fried fish from the Hawassa open fish market and identify the nutritional quality index to show the risk and direction of the management system.

\section{Materials and Methods}

2.1. Description of the Sample Collection Area. Hawassa is the capital city of the southern region and Sidamo regional state of Ethiopia. Hawassa city is $275 \mathrm{~km}$ far from the capital city of Ethiopia (Addis Ababa), and the city is known by its lake called Lake Hawassa, which is about $95 \mathrm{sq} \mathrm{km}$ wide and stretches $16 \mathrm{~km}$ from northeast to southwest and $8 \mathrm{~km}$ from east to west [8]. The lake depth is $21.6 \mathrm{~m}$. Geographically, Lake Hawassa is found in $6033^{\prime}-7033^{\prime} \mathrm{N}$ and $38022^{\prime}-38$ $029^{\prime} \mathrm{E}$. The lake has no outflow and receives inflow from the factory via the Tikur Wuha River [9]. The lake is named yefiker Hayek in Amharic meaning adorable lake. The lake is very useful economically for the entrepreneurs. The lake has more than three fish species, and Nile tilapia (Koroso) is the dominant fish species found in the lake [8]. The lake serves three types of fish products, fillet, fried fish, and fish soup [10].

\subsection{Sample Collection and Preparation. Sampling operation} was carried out during the dry season. Raw fish was collected from Hawassa Lake (Gudumale Park), and the fishes were immediately dissected in the field and cleaned, descaled, and eviscerated manually using a sterile plastic knife [11]. The edible part (flesh and skin) was transferred into plastic bags and kept in an icebox at a temperature of $4^{\circ} \mathrm{C}$ with a fish/ice ratio of $1: 2$ and then transported to the laboratory. The fried fish was randomly collected from the Hawassa fried fish selling market (Fikir Hayik). Raw and fried fishes were grounded to $0.3 \mathrm{~mm}$ size, and the powder was stored in desiccators for analysis. Samples were homogenized by passing them twice through a mincer with $4 \mathrm{~mm}$ holes and mixed thoroughly. Uniformity of the homogenate was ensured by further mixing. The resultant homogenate was packed into several small, convenient sterile containers and stored at $0^{\circ} \mathrm{C}$ until analysis. The experiment was conducted in duplicate for all analyses.

2.3. Determination of Fatty Acid Profiles. The fatty acid profile was determined by using a gas chromatography-mass spectrophotometer (GCMS). Lipid was extracted following the standard procedure in [12]. A homogenized fried fish sample of $5 \mathrm{~g}$ was weighed into a conical flask and dried for $1 \mathrm{hr}$ at $105^{\circ} \mathrm{C}$. The flask was cooled to room temperature, and $50 \mathrm{ml}$ of $4 \mathrm{M}$ hydrochloric acid was added; then, the solution was boiled for $1 \mathrm{hr}$. Water $(150 \mathrm{ml})$ was added, then the solution was filtered by fluted filter paper, and washed until a neutral reaction to litmus paper. The filter paper was dried for $1 \mathrm{hr}$ at $105^{\circ} \mathrm{C}$ and inserted into an extraction thimble of the Soxhlet apparatus. Fat extraction was continued with hexane using a Soxhlet for $6 \mathrm{hr}$. Hexane was evaporated using a rotary evaporator after extraction, and it was kept for derivatization.

Exactly $200 \mathrm{mg}$ of oil was weighed into the bottom of the screw-capped tube (Teflon-lined). $2 \mathrm{ml}$ of $2 \mathrm{~N} \mathrm{KOH}$ was added in methanol. The tube was closed tightly and heated by shaking on a water bath at $80 \underline{0} \mathrm{C}$ for $1 \mathrm{hr}$ and cooled, and then, $5 \mathrm{ml}$ of $5 \% \mathrm{HCl}$ was added in methanol. The tube was closed tightly again and heated by shaking on a water bath at $80^{\circ} \mathrm{C}$ for $1 \mathrm{hr}$. After cooling, $5 \mathrm{ml}$ of hexane and $5 \mathrm{ml}$ of water were added to the tube and mixed, and then, hexane was collected from the layer after short centrifugation. The solvent layer was washed by diluting potassium bicarbonate to remove excess acid and dried over anhydrous sodium or magnesium sulfate. Potassium bicarbonate was recovered after removal of the solvent by evaporation under reduced pressure on a rotary film evaporator. Then, the extract was transferred into a GC vial and injected into it. All the analyses were carried out in triplicates.

2.4. Indexes of Lipid Quality. From fatty-acid composition data, the atherogenicity index and thrombogenicity index were calculated as follows:

Atherogenicity index (AI): it is the relationship between the sum of the main saturated fatty acids and main classes of unsaturated, the former being considered proatherogenic (favoring the adhesion of lipids to cells of the immunological and circulatory system) and the latter being antiatherogenic (inhibiting the aggregation of plaque and diminishing the levels of esterified fatty acid, cholesterol, and phospholipids, thereby preventing the appearance of micro- and macrocoronary diseases [13].

The following equation was applied:

$$
\mathrm{IA}=\frac{[(12: 0+4 \times \mathrm{C} 14: 0+\mathrm{C} 16: 0)]}{\left[\sum \text { MUFAs }+\sum \text { PUFAn6 }+ \text { PUFAn3 }\right)} .
$$

Thrombogenicity index (TI): is the tendency to form clots in blood vessels. It is defined as the relationship between prothrombogenic (saturated) and the antithrombogenic fatty acids (MUFAs, PUFAs $-n 6$, and PUFAs-n3) [13].

$$
\mathrm{IT}=\frac{\mathrm{C} 14: 0+\mathrm{C} 16: 0+\mathrm{C} 18: 0}{0.5 \times \sum \text { MUFAs }+0.5 \times \text { PUFAn6 }+3 \times \text { PUFAn3 }+(\text { PUFAn3/PUFAn6 })}
$$


Hypocholesterolaemic index (h/HI): it is the cholesterol/saturated fat index. Low CSI indicates low saturated fat and cholesterol and low atheroginicity [14].

$\mathrm{hHI}=\frac{(\mathrm{C} 18: 1 \mathrm{n}-9+\mathrm{C} 20: 1(\mathrm{n}-9)+22: 5(n-3)+22: 6(n-3)+\mathrm{C} 18: 3 \mathrm{n}-6+20: 4(n-6)+18: 2(\mathrm{n}-6)+20: 5(n-3))}{\mathrm{C} 14: 0+\mathrm{C} 16: 0}$.

Nutritive value index [15]:

$$
\text { NVI }=\frac{C 18: 0+C 18: 1}{C 16: 0}
$$

Peroxidizability index [16]:

$$
\begin{aligned}
\mathrm{PI}= & \text { mononoic acid } * 0.025+\text { dienoic acid } * 1 \\
& + \text { trienoic acid } * 2+\text { tetranoic acid } * 4 \\
& + \text { pentanoic acid } * 6+\text { hexanoic acid } * 8 .
\end{aligned}
$$

2.5. Data Analyses. The analyses were performed twice for all samples, and closely agreeing replicates were obtained. The data were analyzed using JMP pro 13, one-way ANOVA. The means were separated using Tukey's HSD test at $p<0.05$.

\section{Result and Discussion}

3.1. Assessment of Consumption, Frying, and Marketing of Fish at the Hawassa Lakeside. A preliminary study about the consumption, frying, and marketing of fish at the Hawassa lakeside (Gudumale Park, Tikur wuha, and Fikir Hayik) was conducted. There are more than 18 unions on the lakeside. The preliminary assessments showed more than 60 fishes are fried at the lakeside by each union. Thus, more than 1080 fried fishes are marketable per day. At a time, a maximum of 5 fishes are fried using a metal pan after 5 min of catching, and the frying time is $10 \mathrm{~min}$. The most commonly fried, consumed fish in the lakeside is Nile tilapia (Koroso), and palm oil is the commonly used oil for frying at the lakeside. After frying, all unions put fried fishes in the open air by hanging on wood to collect the oil leaked from fried fish, and the maximum duration of storing fried fishes before serving to customers is $30 \mathrm{~min}$. The unions use the oil several times as they are not well informed about food safety. Thus, the fish can be exposed to many quality and safety degradation.

\subsection{Identified Fatty Acids in Raw and Commercial Fried Fish.} A total of 22 fatty acids were identified in raw and fried Nile tilapia fish muscles (Table 1). Lauric acid (12:0), myristic acid (14:0), pentadecanoic acid (15:0), palmitic acid (16:0), margaric acid $(17: 0)$, stearic acid (18:0), methyl stearate (19:0), arachidic acid (20:0), behenic acid (22:0), and lignoceric acid $(24: 0)$ are the identified saturated fatty acids. Palmitoleic acid (16:1n-7), cis-10-heptadecenoic acid (17: $1 n-10)$, oleic acid (18:1n-9), gondoic acid (20:1n-9), and linoleic acid $(18: 2 n-6)$ are the identified cis-fatty acids. Docosapentaenoic acid $(22: 5 n-3)$, docosahexaenoic acid $(22: 6 n-3)$, and eicosapentaenoic acid $(20: 5 n-3)$ are the identified omega- 3 fatty acids. Linoleic acid $(18: 2 n-6)$, Gamma-linolenic acid (18:3n-6), and arachidonic acid (20: $4 n-6)$ are the identified omega-6 fatty acids. trans-13Octadecenoic acid (18:1n-13) and trans-10-heptadecenoic acid () are the identified trans-fatty acids in raw and commercial fried Nile tilapia fish (koroso).

Among the identified fatty acids, cis-monounsaturated and -polyunsaturated, omega-3, and omega- 6 are the most important contributors to the enrichment of aromatic components [17] and are considered as highly nutritional because of their protective role against cardiovascular diseases and the ability to reduce susceptibility to mental illness, decrease brain and eye function in infants, and alleviate rheumatoid arthritis symptoms [18]. On the contrary, saturated fatty acids cause a rise in the blood cholesterol level as they are easily deposited on the walls of the arteries [19].

\subsection{Fatty Acid Composition of Raw and Commercial Fried Nile} Tilapia Fish (Oreochromis niloticus). The detailed fatty acid profile is presented in (Table 2). Raw and commercial fried fish had ten saturated fatty acids, and the dominant saturated fatty acids in raw and commercial fried fish are palmitic acid (30.92\%-37.01\%), methyl stearate (3.64\%-7.92\%), and myristic acid (1.57\%-2.78\%). Among the identified cis-fatty acids in raw and commercial fried fish, the dominant cisfatty acids are palmitoleic acid $(4.92 \%-1.66 \%)$, oleic acid (38.05\%-33.55\%), and linoleic acid (11.99\%-9.99\%). trans13-Octadecenoic acid and trans-10-heptadecenoic acid are identified as trans-fatty acid, and trans-13-octadecenoic acid is higher which is ranged from $0.12 \%-1.62 \%$. Omega- 3 and omega- 6 fatty acids were also identified in raw and commercial fried Nile tilapia fish. The highest omega-3 fatty acid was decosapentanoic acid varying from $1.08 \%-0.21 \%$, and the highest omega- 6 fatty acid was linoleic acid varying from $11.99 \%-9.99 \%$.

Saturated fatty acids and trans-fatty acids of commercially fried tilapia fish were higher as compared to raw fish. The increase in saturated fatty acids might be because the frying oil is palm oil, and palm oil is high in saturated fat. Similarly, the uncontrolled frying method might result in a high amount of saturated fatty acids in commercially fried fish. Wang et al. [20] stated that a higher temperature can accelerate the reaction rates of oxidation, and more trans-fat could be generated in the frying oil and penetrate the frying fish. A similar finding was reported in [21]. Conversely, cis- 
TABle 1: Identified fatty acids in raw and commercial fried Nile tilapia (Oreochromis niloticus) fish muscles.

\begin{tabular}{|c|c|c|c|}
\hline Formula & Level of saturation & Raw & Commercial fried \\
\hline $12: 0$ & Saturated & $\sqrt{ }$ & $\sqrt{ }$ \\
\hline $14: 0$ & $\sqrt{ }$ & $\sqrt{ }$ & $\sqrt{ }$ \\
\hline $15: 0$ & $\sqrt{ }$ & $\sqrt{ }$ & $\sqrt{ }$ \\
\hline $16: 0$ & $\sqrt{ }$ & $\sqrt{ }$ & $\sqrt{ }$ \\
\hline $17: 0$ & $\sqrt{ }$ & $\sqrt{ }$ & $\sqrt{ }$ \\
\hline $18: 0$ & $\sqrt{ }$ & $\sqrt{ }$ & $\sqrt{ }$ \\
\hline $19: 0$ & $\sqrt{ }$ & $\sqrt{ }$ & $\sqrt{ }$ \\
\hline $20: 0$ & $\sqrt{ }$ & $\sqrt{ }$ & $\sqrt{ }$ \\
\hline $22: 0$ & $\sqrt{ }$ & $\sqrt{ }$ & $\sqrt{ }$ \\
\hline $24: 0$ & $\sqrt{ }$ & $\sqrt{ }$ & $\sqrt{ }$ \\
\hline $16: 1 n-7$ & cis-Monounsaturated & $\sqrt{ }$ & $\sqrt{ }$ \\
\hline $17: 1 n-10$ & $\sqrt{ }$ & $\sqrt{ }$ & $\sqrt{ }$ \\
\hline $18: 1 n-9$ & $\sqrt{ }$ & $\sqrt{ }$ & $\sqrt{ }$ \\
\hline $20: 1 n-9$ & $\sqrt{ }$ & $\sqrt{ }$ & $\sqrt{ }$ \\
\hline $18: 2 n-6$ & cis-Polyunsaturated & $\sqrt{ }$ & $\sqrt{ }$ \\
\hline $18: 1 n-13$ & $\sqrt{ }$ & $\sqrt{ }$ & $\sqrt{ }$ \\
\hline $17: 2(8,10)$ & trans-Monounsaturated & $\sqrt{ }$ & $\sqrt{ }$ \\
\hline $22: 5 n-3$ & Omega-3 & $\sqrt{ }$ & $\sqrt{ }$ \\
\hline $22: 6 n-3$ & $\sqrt{ }$ & $\sqrt{ }$ & $\sqrt{ }$ \\
\hline $20: 5 n-3$ & $\sqrt{ }$ & $\sqrt{ }$ & $\sqrt{ }$ \\
\hline $18: 3 n-6$ & Omega-6 & $\sqrt{ }$ & $\sqrt{ }$ \\
\hline $20: 4 n-6$ & $\sqrt{ }$ & $\sqrt{ }$ & $\sqrt{ }$ \\
\hline
\end{tabular}

TABLE 2: Fatty acid composition of raw and commercial fried fish.

\begin{tabular}{lcc}
\hline Formula & Raw & Commercial fried \\
\hline $12: 0$ & $0.25 \pm 0.01$ & $0.65 \pm 0.05$ \\
$\mathbf{1 4}: \mathbf{0}$ & $\mathbf{1 . 5 7} \pm \mathbf{0 . 1 4}$ & $\mathbf{2 . 7 8} \pm \mathbf{0 . 0 2 5}$ \\
$\mathbf{1 5}: \mathbf{0}$ & $\mathbf{0 . 1 9} \pm \mathbf{0 . 0 1 1}$ & $\mathbf{0 . 4 3} \pm \mathbf{0 . 0 2 1}$ \\
$16: 0$ & $30.92 \pm 0.47$ & $37.01 \pm 1.02$ \\
$17: 0$ & $0.49 \pm 0.03$ & $0.96 \pm 0.012$ \\
$\mathbf{1 8}: \mathbf{0}$ & $\mathbf{0 . 1 1} \pm \mathbf{0 . 0 1 4}$ & $\mathbf{0 . 2 9} \pm \mathbf{0 . 0 1 8}$ \\
$19: 0$ & $3.64 \pm 0.71$ & $7.92 \pm 0.042$ \\
$20: 0$ & $0.68 \pm 0.07$ & $0.69 \pm 0.024$ \\
$22: 0$ & $0.14 \pm 0.02$ & $0.47 \pm 0.028$ \\
$24: 0$ & $0.10 \pm 0.012$ & $0.40 \pm 0.014$ \\
$16: 1 n-7$ & $4.92 \pm 0.56$ & $1.66 \pm 0.72$ \\
$17: 1 n-10$ & $0.75 \pm 0.012$ & $0.10 \pm 0.02$ \\
$18: 1 n-9$ & $38.05 \pm 0.58$ & $33.58 \pm 0.98$ \\
$20: 1 n-9$ & $0.86 \pm 0.08$ & $0.10 \pm 0.04$ \\
$18: 2 n-6$ & $11.99 \pm 1.4$ & $9.99 \pm 0.79$ \\
$18: 1 n-13$ & $0.12 \pm 0.01$ & $1.62 \pm 0.029$ \\
$17: 2(8,10)$ & $0.01 \pm 0.001$ & $0.31 \pm 0.035$ \\
$22: 5 n-3$ & $1.08 \pm 0.014$ & $0.21 \pm 0.012$ \\
$22: 6 n-3$ & $1.02 \pm 0.023$ & $0.20 \pm 0.03$ \\
$20: 5 n-3$ & $0.82 \pm 0.12$ & $0.10 \pm 0.04$ \\
$18: 3 n-6$ & $1.06 \pm 0.04$ & $0.10 \pm 0.03$ \\
$20: 4 n-6$ & $0.87 \pm 0.028$ & $0.10 \pm 0.02$ \\
\hline
\end{tabular}

and essential fatty acids were lower in the fried fish compared to raw tilapia fish. The decrease in cis- and essential fatty acids are associated with the increase in trans- and saturated fatty acids. According to Saguy [22], the increase in trans-fatty acids might be due to the decrease in cis-fatty acids by thermal oxidation, and cis-fatty acids can be converted or isomerized into trans-fatty acids. The increment of saturated fatty acids and trans-fatty acids was supported by a strong significant negative correlation with the decrement of essential fatty acids (omega-3 and omega-6) and cis-fatty acids (\%) at both $\alpha=0.05$ and 0.01 with rcalc $=-1$.

3.4. Nutritional Quality Index (NQI) of Raw and Commercial Fried Fish. Fried fish consumption is popular worldwide including in Ethiopia. Recently, a few studies have been conducted and stated that Nile tilapia fish is the major fish being fried and consumed in the Hawassa open market 
(Ethiopia) [7]. The frying oil used in an open fish market is palm oil because the oil is cheap and provided through cooperatives easily; this oil has been reused for a long time, and this might affect the fatty-acid-related nutritional quality index. Our study showed, in commercially fried fish, the saturated fatty acid, atherogenic index, thrombogenic index, and hypercholesterolemic index have been increased. On contrary to this, the unsaturated fatty acid, hypocholesterolemic index, and nutritive value index have been decreased. Therefore, frying noticeably decreases the nutritional quality of fish muscle. The summation of saturated fatty acids, unsaturated fatty acids including essential, trans-, and cis-fatty acids, and the nutritional quality index is detailed in Table 3.

The total saturated fatty acids, cis-fatty acids, trans-fatty acids, omega- 3 fatty acids, and omega- 6 fatty acids of raw and commercial fried fish were ranging from $38.27-52.07 \%$, $56.57-45.43 \%, 0.13-1.93 \%, 2.92-0.51 \%$, and $13.92-10.19 \%$, respectively. Similarly, the summation of monounsaturated and polyunsaturated fatty acids varied from $44.71-37.37 \%$ and $16.85-10.71 \%$, respectively. The $\sum$ PUFA/SFA and $\sum \omega-3 / \omega-6$ are commonly used parameters to judge the nutritional quality and healthiness of intramuscular fat for human consumption. Kang et al. [23] reported that a balanced intake of dietary $\sum$ PUFA/SFA is very important in regulating serum cholesterol.

Indeed, the ratio of $\sum$ UFA/SFA and $\sum$ PUFA/SFA greater than 0.45 is recommended in human diets for cardiovascular (CVD) and chronic disease prevention [24]. In the meantime, a diet with a ratio of $\sum$ UFA/SFA and $\sum$ PUFA/SFA below 0.45 is considered undesirable for consumption due to its potential to induce cholesterol increase in the blood [25]. The result of this study showed that the $\sum$ UFA/SFA and $\sum$ PUFA/SFA of raw fish are about $1.16 \%$ and $0.44 \%$, which is beneficial for human health. However, the ratio of $\sum$ PUFA/SFA for commercially fried fish is 0.20 which is below the recommended level, and the commercially fried fish is not considered as a balanced human diet and it cannot improve the balance of fatty acids in the tissue. According to Wood et al., 2003, the recent focus of nutritionists is omega- 3 and omega- 6 fatty acids, and the high proportion of PUFA is not necessarily healthy unless it is not balanced with the $\omega-3 / \omega-6$ ratio.

Omega-3, omega- 6 , and the ratio of $\omega-6 / \omega-3$ are principal fatty acids to control the hypocholesterolemic index. Omega-3 and omega- 6 fatty acids regulate the thrombogenic and atherogenic index, respectively. Wolosyzyn et al. [24] reported that healthy meat and meat kind products are characterized by a low atherogenic index and high h/H index. Attia et al. [26] also reported that meat and meat kind products with a low thrombogenicity decrease threat of atrial fibrillation. According to Fernandes et al. [14], if the ratio of $\omega-6 / \omega-3$ is less than 4 , then the diet has a desirable quantity of omega- 3 and omega- 6 fatty acids and reduces cardiovascular diseases. In our study, the ratio of omega- 6 and omega- 3 fatty acids of raw and commercial fried Nile tilapia fish was $4.76 \%$ and $19.98 \%$, respectively, and this showed that raw fish is good and beneficial for human health since the ratio is not as such deviated from the standard. However, the ratio $\omega-6 / \omega-3$ for commercially fried fish is significantly higher than the standard value, and consumption of the commercially fried fish couldnot be beneficial for human health and can expose to noncommunicable diseases.

In addition to the ratio of $\sum$ UFA/SFA, $\sum$ PUFA/SFA, and $\sum \omega-6 / \omega-3$, it is important to evaluate DHA, EPA, atherogenicity, thrombogenicity, nutritive value, and hypocholesterolemic/hypercholesterolemic index $(\mathrm{HH})$ as they indicate the contribution of fish fatty acids to human health. Maki et al. [27] reported that DHA decreases the concentration of low-density lipoprotein cholesterol in plasma and EPA is recognized as the most important essential fatty acid of the $\omega-3$ series in the human diet because it is the precursor to the formation of $\omega-3$ series eicosanoids [28]. In the study, DHA and EPA of raw and commercially fried Nile tilapia fish vary from $1.02-0.25 \%$ and $0.82-0.1 \%$, respectively. The study revealed that raw fish had higher DHA and EPA compared to commercially fried fish. The lower DHA and EPA in commercially fried fish might be because double bonds are highly labile for oxidation during heating, owing to the presence of (pi) bonds, which consequently produces degradable products, though the essential fatty acid would be degraded [29].

3.5. Nutritive Value of Raw and Commercial Fried Fish. The ratio of the nutritive value index of raw and commercial fried fish is shown in (Figure 1). Atherogenicity indicates the adhesion of lipids to cells of the immunological and circulatory system, inhibiting the aggregation of plaque and diminishing the levels of esterified fatty acid, cholesterol, and phospholipids, thereby preventing the appearance of microand macrocoronary diseases, and myristic and palmitic acids are among the most atherogenic agents [30].

Thrombogenicity showed the tendency to form clots in the blood vessels [31]. Stearic is thought to be neutral to atherogenicity but considered to be thrombogenic [32]. According to Estuary et al. [33] and Karimian et al. [34], the higher index of atherogenicity and thrombogenicity values is responsible for the formation of atheroma and stimulates the aggregation of platelets in the cardiovascular system. Hence, the lower values are desirable in the prevention of cardiovascular disorders. The current study showed IA and IT of raw and commercial fried Nile tilapia fish ranged from $0.60-1.01 \%$ and $0.85-1.58 \%$, respectively. This indicates frying leads to increase IA and IT which is considered to be low in nutritional quality.

The index of the ratio between hypocholesterolemic and hypercholesterolemic of fatty acid $(\mathrm{h} / \mathrm{H}$ index) is another indicator of the effect of specific fatty acids on cholesterol metabolism. The higher value of this index is considered to be desirable. Thus, nutritionally higher $\mathrm{h} / \mathrm{H}$ values are considered more beneficial for human health. In the current study, the $\mathrm{h} / \mathrm{H}$ index obtained in raw fish is about 1.81 and the $\mathrm{h} / \mathrm{H}$ index for commercially fried fish is 1.15 . This indicates raw fish is more healthy and beneficial as compared to fried ones. The highest NVI value was found in raw fish 
TABLE 3: Nutritional quality indices of lipids in raw and commercial fried fish.

\begin{tabular}{lcc}
\hline Item & Raw fish & Commercially fried fish \\
\hline$\sum$ SFA & $38.27 \pm 0.04$ & $52.07 \pm 0.07$ \\
$\sum$ MUFA & $44.71 \pm 0.026$ & $37.37 \pm 0.02$ \\
$\sum$ PUFA & $16.85 \pm 0.14$ & $10.7 \pm 0.13$ \\
$\sum$ MUFA/SFA & $1.16 \pm 0.12$ & $0.711 \pm 0.14$ \\
$\sum$ PUFA/SFA & $0.44 \pm 0.001$ & $0.20 \pm 0.001$ \\
$\sum$ omega-3 & $2.92 \pm 0.01$ & $0.51 \pm 0.02$ \\
$\sum$ omega-6 & $13.92 \pm 0.56$ & $10.19 \pm 0.28$ \\
$\sum \omega-3 / \omega-6$ & $0.21 \pm 0.01$ & $0.05 \pm 0.001$ \\
$\sum \omega-6 / \omega-3$ & $4.76 \pm 0.21$ & $19.98 \pm 0.68$ \\
$\sum$ cis & $56.57 \pm 0.015$ & $45.43 \pm 0.03$ \\
$\sum$ trans & $0.13 \pm 0.007$ & $1.93 \pm 0.03$ \\
IA & $0.60 \pm 0.001$ & $1.01 \pm 0.002$ \\
IT & $0.85 \pm 0.02$ & $1.58 \pm 0.21$ \\
h & $55.75 \pm 0.06$ & $44.083 \pm 0.01$ \\
H & $32.49 \pm 0.001$ & $39.79 \pm 0.002$ \\
h/H & $1.71 \pm 0.001$ & $1.10 \pm 0.001$ \\
NVI & $1.23 \pm 0.003$ & $0.91 \pm 0.001$ \\
PI $(\%)$ & $38.27 \pm 0.21$ & $15.45 \pm 0.16$ \\
\hline S & &
\end{tabular}

$\sum$ SFA, saturated fatty acid; $\sum$ MUFA, monounsaturated fatty acid; $\sum$ PUFA, polyunsaturated fatty acid; DHA, docosahexaenoic acid; EPA, eicosapentaenoic acid; IA, atherogenic index; IT, thrombogenic index; $\mathrm{h} / \mathrm{H}$, hypocholesterolemic/hypercholesterolemic index; NVI, nutritive value index; PI, peroxidizability index.

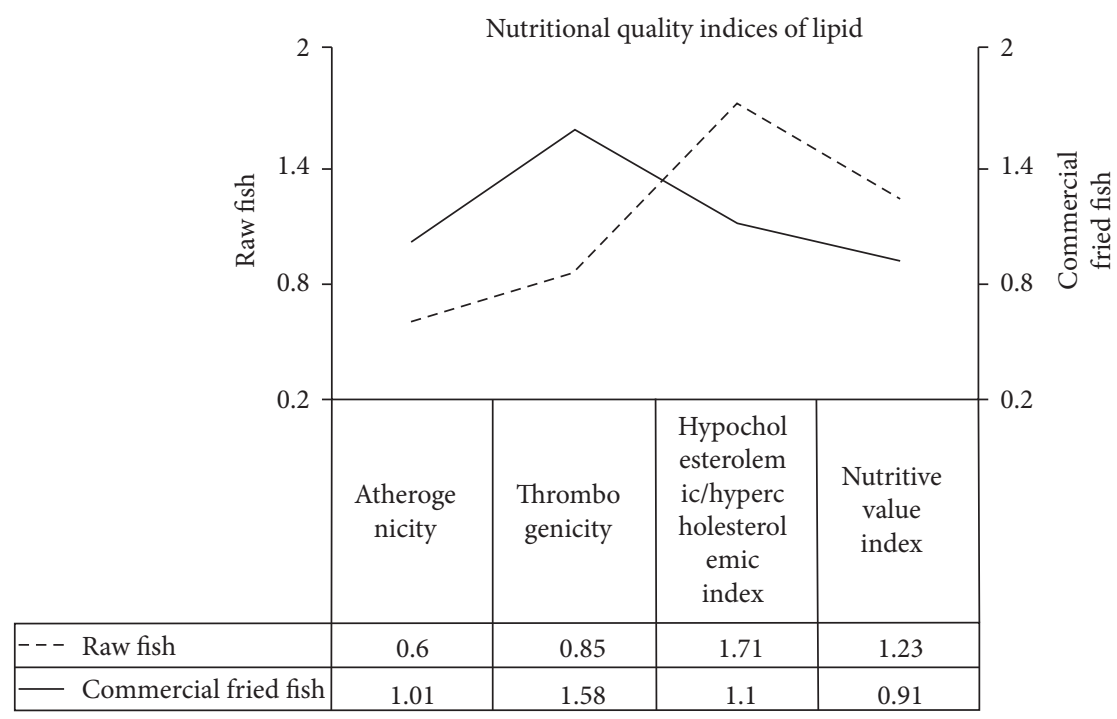

FIGURE 1: Nutritive value index of raw and commercial fried fish.

which about 1.23 as compared to commercially fried Nile tilapia fish whose NVI value is 0.91 . The highest NVI value in raw fish is because raw fish had the highest proportion of $\mathrm{C}$ $18: 0, \mathrm{C} 18: 1 n-9$, and the lowest percentage of C $16: 0$.

3.6. Comparison of the Hypocholesterolemic and Peroxidizability Index of Raw and Commercial Fried Fish. The hypocholesterolemic, hepercholestrolemic, and peroxidizability index of raw and commercial fried fish are shown in Figure 2. Peroxidizability index (PI) is another parameter to indicate the relationship between the fatty acid composition of a tissue and its susceptibility to oxidation. The PI index assesses the stability of PUFA included in food products and protects from possible oxidation. The higher PI value is equivalent to the greater protective potential for coronary artery disease. According to Kang et al. [23], Sinanoglou et al. [35], and Skalecki et al. [36], excessive intake of PUFA leads to oxidative stress because lipid is susceptible to lipid peroxidation. Oxidative stress is associated with the formation of lipid peroxides, and has been suggested as contributing to pathological processes in aging and many diseases such as atherosclerosis.

In the current study, the PI value of raw and commercial fried fish ranged from $38.27 \%-15.45 \%$. Thus, the study revealed that commercially fried Nile tilapia fish had a lower PI value compared to raw fish, and the lower PI value indicates low level of PUFA for auto-oxidation. However, raw 


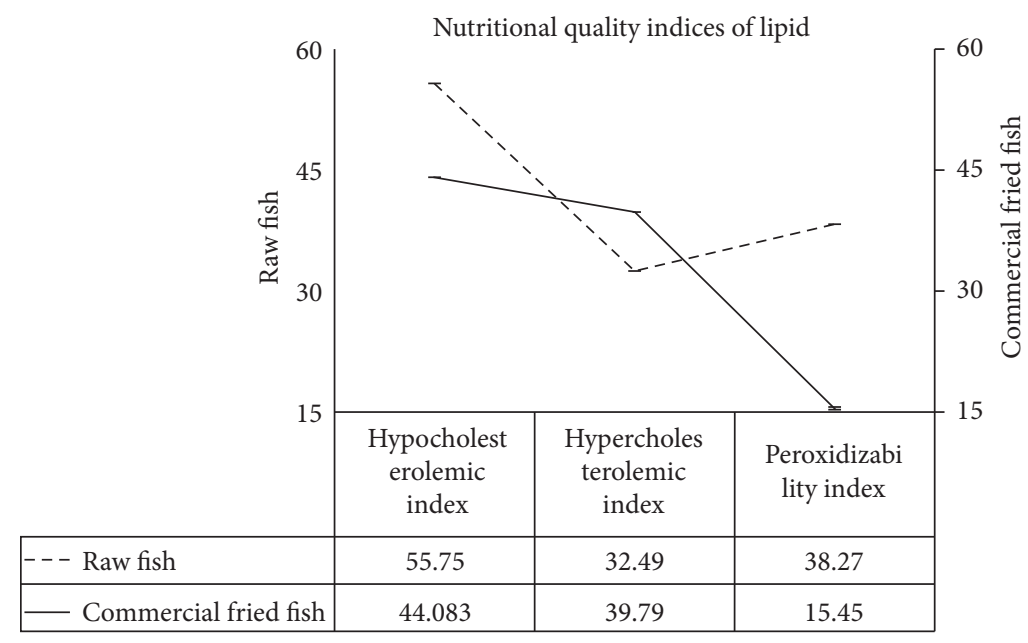

FIgURE 2: Hypocholesterolemic and peroxidizability index of raw and commercial fried fish.

fish had high PUFA and susceptible to auto-oxidation. The higher tendency to lipid oxidation was found in different kinds of marine fish fillet by Ghaeni et al. [31] and in crab edible tissue by Fernandes et al. [14].

\section{Conclusions and Recommendations}

The dominant saturated fatty acids found in Nile tilapia fish were palmitic, myristic acid, and methyl stearate. Oleic acid, linoleic acid, and palmitoleic acid were the highest cis-fatty acids. Saturated and trans-fatty acids were correlated positively. Similarly, unsaturated and cis-fatty acids were found to have a positive correlation. Regarding the nutritional quality index, raw fish was found with a high amount of essential fatty acids, $\mathrm{h} / \mathrm{H}$ index, nutritive value index, and peroxidizability index which is considered beneficial for human health. Conversely, in the commercially fried fish, a high amount of IA and IT was found, which could have a negative influence on human health. Thus, it could be recommended to set the frying time and temperature during fish frying, and further analysis should be conducted about the frying oil's effect on the nutritional composition of fried fish.

\section{Data Availability}

The datasets used and/or analyzed during the current study are available from the corresponding author on reasonable request.

\section{Conflicts of Interest}

The authors declare that there are no conflicts of interest regarding authorship and/or publication of this article.

\section{Authors' Contributions}

Aemiro Tadesse is the principal investigator, designed the study, took part in the sample collection process and laboratory experiment, entered and analyzed the data, and wrote the manuscript of the current study. Derese Tamiru oversaw the whole sample collection process, giving comments and suggestion during the manuscript write up. The authors read and approved the final manuscript to be submitted.

\section{Acknowledgments}

The current study was funded by Hawassa University as a part, and the authors are grateful for the fund and help. The donors had no direct involvement in data collection, analysis, interpretations, and experimental work. The authors also acknowledge the School of Nutrition, Food Science, and Technology of Hawassa University for support given during the sample collection processes.

\section{References}

[1] A. Jayedi and S. Shab-Bidar, "Fish consumption and the risk of chronic disease: an umbrella review of meta-analyses of prospective cohort studies," Advances in Nutrition, vol. 11, no. 5, pp. 1123-1133, 2020.

[2] K. S. Leung, J. Galano, T. Durand, and J. C. Lee, "Profiling of omega-polyunsaturated fatty acids and their oxidized products in salmon after different cooking methods," Antioxidants, vol. 7, no. 8, p. 96, 2018.

[3] T. Gadiraju, Y. Patel, J. Gaziano, and L. Djoussé, "Fried food consumption and cardiovascular health: a review of current evidence," Nutrients, vol. 7, no. 10, pp. 8424-8430, 2015.

[4] E. K. Dery, E. E. Carey, R. T. Ssali et al., "Sensory characteristics and consumer segmentation of fried sweet potato for expanded markets in Africa," International Journal of Food Science \& Technology, vol. 56, no. 12472, 2020.

[5] E. K. Oke, M. A. Idowu, O. P. Sobukola, S. A. O. Adeyeye, and A. O. Akinsola, "Frying of food: a critical review," Journal of Culinary Science \& Technology, vol. 16, no. 2, pp. 107-127, 2018.

[6] FAO, Assessment and Management of Seafood Safety and Quality: Current Practices and Emerging Issues, FAO, Rome, Italy, 2014.

[7] A. Tadesse, "Proximate composition, minerals, and sensory acceptability of deep fried nile Tilapia fish (Oreochromis niloticus) as influenced by repeated use of palm oil," Food Science and Quality Management, vol. 95, pp. 19-28, 2020. 
[8] N. Kebtieneh, Y. Alemu, and M. Tesfa, "Stock assessment and estimation of maximum sustainable yield for Tilapia stock (oriocromis niloticus) in lake Hawassa, Ethiopia," Agriculture, Forestry and Fisheries, vol. 5, no. 4, pp. 97-107, 2016.

[9] K. Gebremedhin and T. Berhanu, "Determination of some selected heavy metals in fish and water samples from Hawassa and Ziway Lakes," Science Journal of Analytical Chemistry, vol. 3, no. 1, pp. 10-16, 2015.

[10] A. Lema Abelti, "Minerals content and fatty acids profile of nile Tilapia (Oreochromis niloticus) fillet from lake zeway: effect of endogenous factors," Journal of Nutrition \& Food Sciences, vol. 7, no. 1, pp. 7-9, 2017.

[11] J. M. Njinkoue, I. Gouado, F. Tchoumbougnang et al., "Proximate composition, mineral content and fatty acid profile of two marine fishes from Cameroonian coast: pseudotolithus typus (Bleeker, 1863) and Pseudotolithus elongatus (Bowdich, 1825)," NFS Journal, vol. 4, pp. 27-31, 2016.

[12] M. Petrovic, N. Kezić, and V. Bolanča, "Optimization of the GC method for routine analysis of the fatty acid profile in several food samples," Journal of Food Chemistry, vol. 122, no. 1, pp. 285-291, 2010.

[13] L. Senso, M. D. Suárez, T. Ruiz-Cara, and M. García-Gallego, "On the possible effects of harvesting season and chilled storage on the fatty acid profile of the fillet of farmed gilthead sea bream (sparus aurata)," Food Chemistry, vol. 101, no. 1, pp. 298-307, 2007.

[14] C. E. Fernandes, M. A. D. S. Vasconcelos, M. De Almeida Ribeiro, L. A. Sarubbo, S. A. C. Andrade, and A. B. D. M. Filho, "Nutritional and lipid profiles in marine fish species from Brazil," Food Chemistry, vol. 160, pp. 67-71, 2014.

[15] Y. Chen, Y. Qiao, Y. Xiao et al., "Differences in physicochemical and nutritional properties of breast and thigh meat from crossbred chickens, commercial broilers, and spent hens," Asian-Australasian Association of Animal Production Societies, vol. 29, pp. 855-864, 2016.

[16] M. C. Erickson, "Variation of lipid and tocopherol composition in three strains of channel catfish (Ictalurus punctatus)," Journal of the Science of Food and Agriculture, vol. 59, no. 4, pp. 529-536, 1992.

[17] J. S. Elmore, D. S. Mottram, M. Enser, and J. D. Wood, "Effect of the polyunsaturated fatty acid composition of beef muscle on the profile of aroma volatiles," Journal of Agricultural and Food Chemistry, vol. 47, no. 4, pp. 1619-1625, 1999.

[18] G. Hornstra, "Lipids in functional foods in relation to cardiovascular disease," Lipid-Fett, vol. 101, no. 12, pp. 456-466, 1999.

[19] M. A. Garaffo, R. Vassallo-Agius, Y. Nengas et al., "Fatty acids profile, atherogenic (IA) and thrombogenic (IT) health lipid indices, of raw roe of blue fin tuna (Thunnus thynnus L.) and their salted product "bottarga"," Food and Nutrition Sciences, vol. 2, no. 7, pp. 736-743, 2011.

[20] Y. Wang, T. Hui, Y. W. Zhang et al., "Effects of frying conditions on the formation of heterocyclic amines and trans fatty acids in grass carp (Ctenopharyngodon idellus)," Food Chemistry, vol. 167, pp. 251-257, 2015.

[21] Y. Moradi, J. Bakar, A. A. Motalebi, S. H. Syed Muhamad, and Y. Che Man, "A review on fish lipid: composition and changes during cooking methods," Journal of Aquatic Food Product Technology, vol. 20, no. 4, pp. 379-390, 2011.

[22] I. S. Saguy and D. Dana, "An integrated approach to deep fat frying: engineering, nutrition, health, and consumer aspects,"
Journal of Food Engineering, vol. 56, no. 2-3, pp. 143-152, 2003.

[23] M. J. Kang, M. S. Shin, J. N. Park, and S. S. Lee, "The effects of polyunsaturated:saturated fatty acids ratios and peroxidisability index values of dietary fats on serum lipid profiles and hepatic enzyme activities in rats," British Journal of Nutrition, vol. 94, no. 4, pp. 526-532, 2005.

[24] J. Wołoszyn, G. Haraf, A. Okruszek, M. Wereńska, Z. Goluch, and M. Teleszko, "Fatty acid profiles and health lipid indices in the breast muscles of local Polish goose varieties," Poultry Science, vol. 99, no. 2, pp. 1216-1224, 2020.

[25] C. Mapiye, M. Chimonyo, K. Dzama, A. Hugo, P. E. Strydom, and V. Muchenje, "Fatty acid composition of beef from Nguni steers supplemented with Acacia karroo leaf-meal," Journal of Food Composition and Analysis, vol. 24, no. 4-5, pp. 523-528, 2011.

[26] Y. A. Attia, M. A. Al-Harthi, M. A. Korish, and M. M. Shiboob, "Fatty acid and cholesterol profiles, hypocholesterolemic, atherogenic, and thrombogenic index of broiler meat in the retail market," Lipids in Health and Disease, vol. 16, no. 40, pp. 1-11, 2017.

[27] K. C. Maki, M. E. Van Elswyk, D. McCarthy et al., "Lipid responses to a dietary docosahexaenoic acid supplement in men and women with below average levels of high density lipoprotein cholesterol," Journal of the American College of Nutrition, vol. 24, no. 3, pp. 189-199, 2005.

[28] I.-C. Chen, F. A. Chapman, C.-I. Wei, K. M. Porteir, and S. F. O'Keefe, "Differentiation of cultured and wild sturgeon (Acipenser oxyrinchus desotoi) based on fatty acid composition," Journal of Food Science, vol. 60, no. 3, pp. 631-635, 1995.

[29] A. U. Türkkan, S. Cakli, and B. Kilinc, "Effects of cooking methods on the proximate composition and fatty acid composition of seabass (Dicentrarchus labrax, Linnaeus, 1758)," Food and Bioproducts Processing, vol. 86, no. 3, pp. 163-166, 2008.

[30] V. Laudadio and V. Tufarelli, "Growth performance and carcass and meat quality of broiler chickens fed diets containing micronized-dehulled peas (Pisum sativum cv. Spirale) as a substitute of soybean meal," Poultry Science, vol. 89, no. 7, pp. 1537-1543, 2010.

[31] M. Ghaeni and K. N. Ghahfarokhi, "Fatty acids profile, atherogenic (IA) and thrombogenic (IT) health lipid indices in leiognathusbindus and upeneussulphureus," Journal of Marine Science: Research \& Development, vol. 3, no. 4, pp. 3-5, 2013.

[32] I. I. Vitina, S. Cerina, J. Jansons et al., "Functional poultry meat enriched with biologically active substances from neutral extractives obtained from spruce needles," Krmiua, vol. 54, no. 5, pp. 151-158, 2012.

[33] P. R. Estuary, X. Z. Id, X. Ning, X. He, X. Sun, and X. Yu, "Fatty acid composition analyses of commercially important fish species from the pearl river estuary," PLoS One, vol. 15, no. $1,2020$.

[34] N. Karimian-Khosroshahi, H. Hosseini, M. Rezaei, R. Khaksar, and M. Mahmoudzadeh, "Effect of different cooking methods on minerals, vitamins, and nutritional quality indices of rainbow trout (Oncorhynchus mykiss)," International Journal of Food Properties, vol. 19, no. 11, pp. 2471-2480, 2016.

[35] V. J. Mahmoudzadeh, A. Batrinou, F. Mantis, I. Bizelis, and S. Miniadis-Meimaroglou, "Lipid quality indices: differentiation of suckling lamb and kid breeds reared by traditional 
sheep farming," Small Ruminant Research, vol. 113, no. 1, pp. 1-10, 2013.

[36] P. Skalecki, M. Florek, A. Pyc, A. Kaliniak, and A. Staszowska, "Comparison of physicochemical properties, fatty acid composition, and mineral contents in common carp (Cyprinus carpio $\mathrm{L}$ ) fillet and the native traditional product carp ham," Polish Journal of Food and Nutrition Sciences, vol. 66, pp. 311-319, 2016. 